CASE REPORT

\title{
Post Arthrotomy Prevotella bivia Septic Arthritis and Secondary Osteomyelitis
}

\author{
Azhar Farooqui ${ }^{1}$, Samer Salih ${ }^{2}$, Abdelmageed Kambal ${ }^{3}$ \\ ${ }^{1}$ Department of Acute Medicine, University Hospitals of Derby and Burton, Derby, UK \\ ${ }^{2}$ Department of Internal Medicine, Suleiman Al Habib Medical Group, Riyadh, Saudi Arabia \\ ${ }^{3}$ Department of Microbiology, Suleiman Al Habib Medical Group, Riyadh, Saudi Arabia
}

\begin{abstract}
Prevotella is a common gram-negative anaerobic rod that is frequently associated with female genitourinary tract infections, pelvic inflammatory disease, aspiration pneumonia, lung abscess and pleural empyema. Osteomyelitis in association with septic arthritis is rare with only a handful of cases reported worldwide. In this case study, we report a patient who presented with a clinical picture of septic arthritis complicated by the presence of a sinus tract at the site of a previous arthroscopic surgery. Initial sinus pus cultures demonstrated Streptococcus agalactiae, which were confirmed with intra-operative joint fluid cultures. After a brief period of clinical improvement following arthrotomy, a sudden clinical deterioration prompted re-cultures demonstrating heavy growth of Provetella bivia. Repeat MRI demonstrated new signs of osseous involvement. The patient was managed with a redo arthrotomy, extensive debridement of osteomyelitic tissue, washout and an extended antibiotic therapy regimen. This association is first of its kind in Saudi Arabia. J Microbiol Infect Dis 2019; 9(2):100-103.
\end{abstract}

Keywords: Osteomyelitis, septic arthritis, infectious disease, Prevotella, arthrotomy

\section{INTRODUCTION}

Prevotella bivia is a gram-negative, beta lactamase producing anaerobic bacilli, previously identified as Bacteroides bivius [1]. It is commonly associated with female genitourinary tract infections where an inverse relationship between its colonization and the absence of hydrogen peroxide producing lactobacilli has been well established $(2,3]$. However, its association with septic arthritis and osteomyelitis is extremely rare with only a handful of cases reported worldwide [4-7].

In this article, the author's describe a case of septic arthritis with Streptococcus agalactiae requiring arthrotomy. After a brief phase of clinical improvement, sudden deterioration prompted bacteriological cultures demonstrating heavy growth of $P$. bivia while repeat imaging demonstrated new signs of osteomyelitis. Patient was managed with a redo surgical debridement and an extended period of antibiotic therapy with good clinical outcome. A brief literature review is also provided.

\section{CASE REPORT}

A 53-year-old woman, known insulin dependent diabetic, presented to our emergency department complaining of right knee pain. Her history started around 6-7 months back, when she was diagnosed with a right knee septic arthritis following a traumatic injury. At that time the wound culture demonstrated Methicillin resistant Staphylococcus aureus (MRSA) requiring intravenous antibiotics for 6 weeks in addition to arthroscopic knee washout and debridement. As this was done at her local hospital, detailed medical record of the episode could not be obtained. As per the patient, the wound had thereafter healed fully and she was completely asymptomatic.

One week prior to current emergency department presentation, patient started to have symptoms of severe, progressive right knee pain, decreased mobility, associated with an appearance of a discharging sinus. This was

Correspondence: Dr. Azhar Farooqui, Department of Acute Medicine, University Hospitals of Derby and Burton, Derby, UK E-mail: amfarooqui91@gmail.com

Received: 23 March 2019 Accepted: 10 April 2019

Copyright (C) JMID / Journal of Microbiology and Infectious Diseases 2019, All rights reserved 
preceded by a mild non-penetrating local trauma to the knee; however, the patient insisted it was unrelated to the cause of her symptoms. Physical examination demonstrated right knee joint redness, effusion and a discharging sinus producing large amounts of green foul smelling pus. Lab analysis demonstrated borderline leukocytosis, in addition to raised acute phase reactants (C-reactive protein of 279 , normal laboratory range is $<5$ ). Kidney functions, and liver functions were within normal. Patient was admitted as a case of septic arthritis for further investigation and management. During her initial assessment, endocrine services were also involved for uncontrolled blood sugar requiring insulin dose adjustments.

MRI was further suggestive of septic arthritis (Image 1) of the right knee with marked knee joint effusion, and the presence of a sinus tract, connecting the skin at the lateral aspect of the knee. Infected Baker's cyst, moderate degenerative changes of the anterior cruciate ligament, patellar tendon and lateral collateral ligament were also observed. No osseous involvement of the infection was observed. After sending samples to the laboratory for bacteriological cultures, patient was initially started on Ceftriaxone and Teicoplanin. Patient underwent right knee arthrotomy, washout and extensive debridement. A moderate amount of frank pus came through the quads tendon incision; the knee was then thoroughly debrided with a lot of synovium and other soft tissues that seemed infected or of ambiguous vascularity removed. Interestingly, the sinus exit to the skin seemed to coincide with the lateral portal of the previous arthroscopy.

Initial culture results from the wound discharge demonstrated Streptococcus agalactiae. Initial blood culture at admission was negative for any growth. Repeat wound culture, which was taken intra-operatively during the arthrotomy confirmed Streptococcus agalactiae. Post procedure, inflammatory markers had a downward trend, with no report of fever post-operatively.

One week post-surgery, patient started to spike fever, with increasing amount of pus discharge from the operative site, with laboratory investigations demonstrating rising inflammatory markers. Cultures from the pus discharge were re-taken. Antibiotics were adjusted to piperacillin/tazobactam and Moxifloxacin.

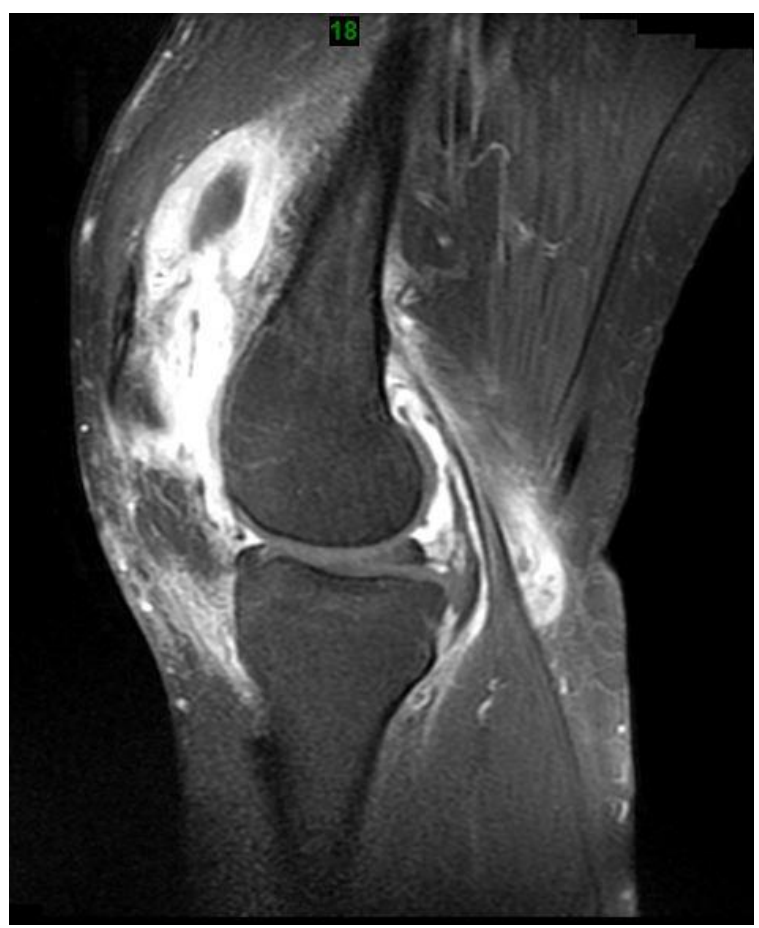

Figure 1. MRI T1 Weighted Image + Fat Suppression: On contrast administration, there is vivid, thick linear contrast enhancement involving the joint fluid as well as the Baker's cyst representing large chronic abscess formation. Also there is moderate diffuse soft tissue edema surrounding the knee joint showing moderate contrast enhancement denoting extension of the inflammation.

Repeat cultures demonstrated heavy growth of $P$. bivia after incubation anaerobically at $37{ }^{\circ} \mathrm{C}$. The organism was sensitive to Imipenem, Meropenem, Metronidazole and Moxifloxacin. Repeated knee MRI (Image 2) demonstrated septic arthritis resulting in distension of joint space due to debrinous joint effusion with early acute secondary osteomyelitis in lateral aspects of both femoral condyles, femoral subchondral region, in posterior aspect of lateral condyle of tibia, both tibial plaetues and proximal end of fibula. Patient underwent right knee soft tissue debridement, excision of osteomyelitic lesion on the medial tibial plateau. The other MRI suggestions of osteomyelitic lesions were ruled out intra-operatively and did not require extensive debridement. Patient completed around 16 weeks of antibiotic therapy. Outpatient follow-up visits demonstrated normal inflammatory markers; complete healing of the knee wound, and improved mobility at the knee joint. 


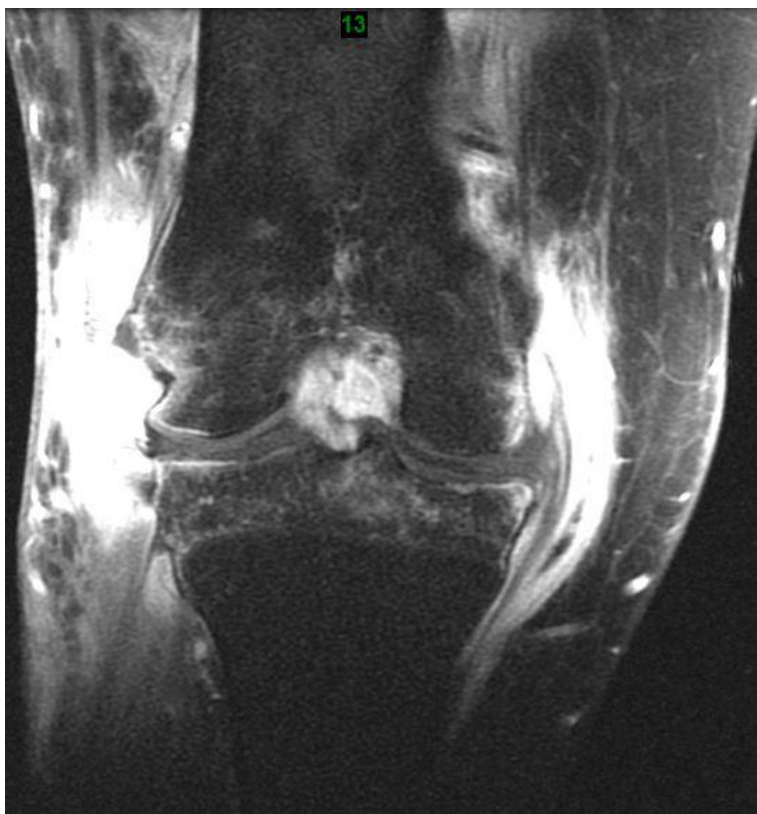

Figure 2. MRI T1 Weighted Image + Fat Suppression: Altered high signal noted in lateral aspects of both femoral condyles, sub-chondral region, in the posterior aspect of the lateral condyle of the tibia, both tibial plaetus suggestive early acute osteomyelits. Joint capsule is bulging with debrinous joint effusion.

\section{DISCUSSION}

$P$. bivia associated septic arthritis is rare. It commonly involves patients with prior joint instrumentation, joint replacement, or presence of pre-existing rheumatologic disease requiring immunosuppressive therapy [4,5,7]. Laiho et al. described a case of $P$. bivia septic arthritis in a patient with juvenile rheumatoid arthritis following an intra-articular glucocorticoid injection in the hip joint [4]. Meanwhile, AlegreSancho et al. described a case of $P$. bivia in a patient with severe, long lasting rheumatoid arthritis treated with low doses of corticosteroids [7]. In both these cases, immunosuppression associated with the underlying autoimmune arthropathy or its subsequent treatment with corticosteroids was postulated as possible risk factors for the development of the septic arthritis. Very rarely, it has also been observed to occur in joints with no pre-existing conditions [6]. In our case however, in an otherwise immune-competent individual, not receiving any corticosteroid therapy, the development of $P$. bivia infection raises the possibility of alternate risk factors. The authors postulate that an initial streptococcus septic arthritis resulted in the formation of a sinus tract at the site of previous arthroscopy; this further aided acute knee inoculation with $P$. bivia resulting in worsening septic arthritis and development of new osteomyelitis. Although metronidazole is commonly used for eradicating this bacterium, we preferred to continue piperacillin/tazobactam and Moxifloxacin, initiated prior to culture results, with favorable clinical response.

The association of Prevotella in obstetrics and gynecological infections is well documented $[2,3]$. Male genitourinary tract has also been reportedly affected; Nalmas et al. [8) reported $P$. bivia causing a penile abscess requiring surgical drainage. Sagristà et al. [9] described a case of inguinal bubo in a young male acquired as part of sexually transmitted infection. Other rare case reports secondary to $P$. bivia include Necrotising Fasciitis [10], abdominal wall phlebitis following renal transplantation and inferior vena cava obstruction [11], chest wall abscess [12], and $P$. bivia associated Lemierre Syndrome [13].

\section{ACKNOWLEDGMENTS}

Conflicting Interests: The authors declare that they have no conflict of interest.

Funding source: None declared.

\section{REFERENCES}

1. Mikamo H, Kawazoe K, Izumi K, et al. Studies on the clinical implications of anaerobes, especially Prevotella bivia, in obstetrics and gynecology; $\mathrm{J}$ Infect Chemother 1998; 4: 177.

2. Hill GB. Anaerobic Flora of the Female Genital Tract. In: Lambe DW, Genco RJ, Mayberry-Carson KJ (eds). Anaerobic Bacteria. Springer, 1980, Boston, MA, USA

3. Sharma H, Tal R, Clark NA, Segars JH. Microbiota and pelvic inflammatory disease.Semin Reprod Med 2014; 32(1):43-49.

4. Laiho K, Kotilainen $P$. Septic arthritis due to Prevotella bivia after intra-articular hip joint injection. Joint Bone Spine 2001; 68(5):443-444.

5. Nseir B, Cutrona A. Prosthetic septic arthritis secondary to Prevotella bivia bacteremia in a patient with polymyalgia rheumatica. Infect D Clin Pract 2008; 16(3):190-191.

6. Salman SA, Baharoon SA. Septic arthritis of the knee joint secondary to Prevotella bivia. Saudi Med J 2009; 30(3):426-428.

7. Alegre-Sancho JJ, Juanola X, Narvaez FJ, RoigEscofet D. Septic arthritis due to Prevotella bivia in a patient with rheumatoid arthritis. Joint Bone Spine 2000; 67(3):228-229. 
8. Nalmas S, Bishburg E, Chan T. Streptococcus constellatus and Prevotella bivia penile abscess. Scientific World J 2007; 7:1631-1633.

9. Sagristà M, Martin-Ezquerra G, Gallardo F, et al. Inguinal syndrome secondary to Prevotella bivia after accidental bite in orogenital sex. Sex Transm Infect 2012; 88:250-251.

10. Lepivert JC, Guinet V, Auquit-Auckbur I. Necrotizing Fasciitis of the Hand and Wrist Due to Prevotella bivia. J Hand Surg 2014; 40 (7):757-758.

11. Janssen S, van Donselaar-van der Pant KA, van der Weerd NC, et al. Abdominal Wall Phlebitis Due to Prevotella bivia Following Renal Transplantation in a Patient with an Occluded Inferior Vena Cava. Infection 2013; 41(1):271-274.

12. Hsu GJ, Chen CR, Lai MC, Luh SP. Chest Wall Abscess Due to Prevotella bivia. J Zhejiang Univ Sci B 2009; 10(3):233-6.

13. Huits RM, van Assen S, Wildeboer-Veloo AC, Verschuuren EA, Koeter GH. Prevotella bivia necrobacillosis following infectious mononucleosis. J Infect 2006; 53(2):e59-e63. 